
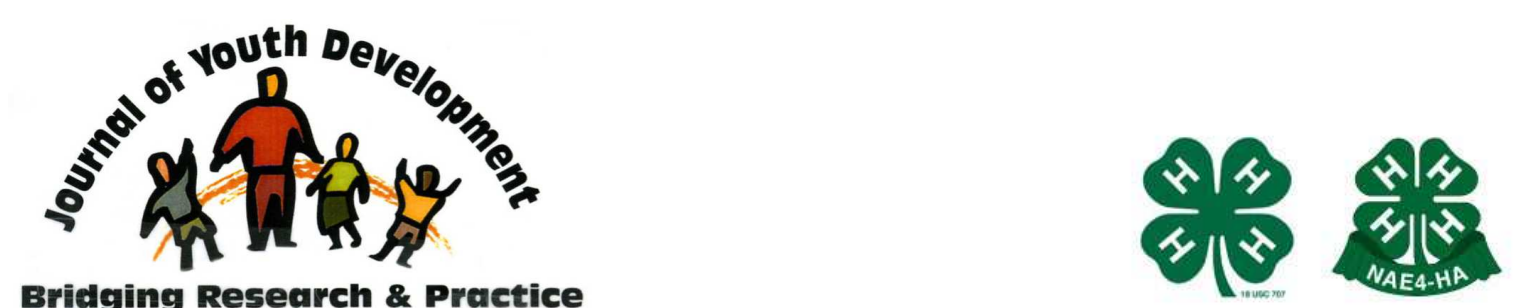

\title{
Nebraska 4-H Household Technology and Interest Survey
}

\author{
Bradley S. Barker, Ph.D. \\ University of Nebraska-Lincoln \\ (402) 472-9008 \\ Fax (402) 472-9024 \\ 114 Agricultural Hall \\ Lincoln, NE 68583-0700 \\ bbarker@unl.edu \\ Date of Submission 1/26/06
}

\author{
Debra K. Meier \\ Nebraska 4-H \\ 114 Agricultural Hall \\ Lincoln, NE 68583-0700 \\ dmeier1@unl.edu
}




\title{
JOURNAL OF YOUTH DEVELOPMENT \\ bridging research and practice

\section{Nebraska 4-H Household Technology and Interest Survey}

\author{
Bradley S. Barker, Ph.D. and Debra K. Meier \\ University of Nebraska-Lincoln
}

\begin{abstract}
Nationally, 4-H has placed renewed emphasis in the areas of Science and Technology as a way to prepare youth for the $21^{\text {st }}$ century workplace. Home access may become necessary to youth as they develop science and technology literacy via 4-H programs. A survey was sent to a random sample of 1,414 Nebraska families from a total population of 13,516 . The survey examined the percentage of families that have access to computers and the Internet at home, computer components, use characteristics and specific areas of interest in science and technology. Results indicate that 96 percent of Nebraska 4-H families have access to computers at home. Nearly 92 percent of families had a connection to the Internet with a majority using dial-up connections. Families are interested in technology programs focused on basic computer knowledge and office application. In science, 4-H families indicated environment sciences and botany were areas of interest.
\end{abstract}

\section{Introduction}

Nationally, 4-H has placed renewed emphasis in the areas of Science and Technology as a way to prepare youth for the $21^{\text {st }}$ century workplace (The National 4-H Strategic Directions Team, 2001). In addition, new program delivery methods utilizing computers and the Internet have obtained increased importance in 4-H curriculum development, programming, and communications. 
Therefore, participation in 4-H program areas will most likely require access to computers and to some extent the Internet. For example, the National 4-H Cooperative Curriculum Systems 2006 Geospatial curriculum will be delivered on four CD-ROMs with supplemental materials available via a website.

While over $92 \%$ of children have access to computers at schools their time may be limited since resources are shared with other students (U.S. Census Bureau, 2003). As a consequence, home access may become increasingly important to children as they develop science and technology literacy via 4-H programs.

According to the U.S. Census Bureau (2003) more than 39 percent of households do not have a computer and 45 percent do not have Internet access at home. The diversification of 4-H curriculum into science and technology program areas and the use of electronic delivery methods necessitate the examination of computer and Internet accessibility in 4-H households.

\section{Purpose}

The purpose of the study was to investigate what technologies Nebraska 4-H families have in their home. In addition, the study was conducted to identify areas of science and technology 4-H families thought were important. The objectives were to:

1) Determine the percentage of families that have access to computers at home and inventory the current state of their technology and describe computer use characteristics.

2) Determine the percentage of families that have access to the internet and determine potential barriers to access.

3) Determine specific areas of interest in science and technology as measured by a selfreported interests inventory

\section{Population}

\section{Procedure}

A random sample of 1,414 families out of a total population of 13,516 Nebraska 4-H families, was selected from the 2004 4-H Plus database. Randomly selected families were sent the paper-based survey via US mail with a pre-paid return envelope. A postcard was mailed approximately two weeks before the survey was mailed to inform selected families of the upcoming study. Follow-up postcards were sent after two, four and six weeks to participants who had not returned the survey.

\section{Instrument}

A 19-question survey was developed based on the U.S. Census Bureau's Computer and Internet Use in the United States: 2003 survey instrument. The survey consisted of 19 questions with a variety of response scales including yes/no questions, 4-point Likert-type scales and one openended question.

To begin, respondents were asked if they had a computer, if not they skipped to question 12 of the survey. Questions 2 through 11 of the survey explored topics pertaining to computers such as operating system, year purchased, components and Internet access. Question 12 asked the main reason for not having a computer. Questions 16 and 18 utilize a 4-point Likert-type scale for questions regarding the priority of different technology and science areas where $1=$ not a priority and a $4=$ high priority. In questions 17 and 19 respondents were asked to rank the first, second and third most important technology and science areas. 


\section{Content Validity and Reliability}

The overall response rate to the survey was 33.6 percent. The confidence interval at the $95 \%$ confidence level is 4.41 indicating that the responses are accurate $95 \%$ of the time plus or minus 4.41 points from the reported mean.

Since the majority of the survey comes from the U.S. Census, the questions have been pretested and reviewed by experts and therefore are deemed to be valid. The results of a Cronbach alpha test for homogeneity of the 19 item instrument revealed a very high standardized alpha coefficient $(r=.96)$. The high reliability coefficient indicates that the test halves are highly correlated and the questionnaire has high internal consistency.

To address the potential of non-response error, the initial respondents were differentiated into two groups. The first group, early respondents, consisted of respondents that returned their surveys from April to the end of June, 2005. The second group, late respondents, consisted of respondents that returned the survey on July 1, 2005 up to the indicated due date. An independent samples $t$ test was conducted to determine if there were any significant differences between the mean scores of early and late groups based on each question. No significant differences were found between the groups on any question in the survey including the existence of a computer in the household $\mathrm{t}(20.66)=-.938, \mathrm{p}=.359$, equal variances not assumed.

In addition, a random sample of 100 additional surveys was sent to the initial group's non respondents to determine if scores were significantly different than the initial respondents. Fifteen surveys were returned by the second-round respondents for a response rate of 15 percent. Due to low statistical power, the second round respondents were combined with the late respondents to create a new group with a sample size of 34 (Linder, Murphy \& Briers, 2001).

Additional independent samples test were conducted to determine if there were any significant differences between the mean scores of early respondents and the combined group of late respondents and second round respondents on the existence of a computer in the household and high-speed internet. No significant differences were found between the groups on the existence of a computer in the household $\mathrm{t}(486)=.703, \underline{p}=.482$ or the use of high-speed Internet access $\mathrm{t}(468)=-1.39, \mathrm{p}=.166$.

\section{Results}

\section{Computer Characteristics}

Overall, $96.4 \%$ of respondents said they had a computer at home. A majority of respondents use Windows XP (57.5\%), followed by Windows 98 (21.9\%) and Windows $2000(10.5 \%)$ see Table 1. Close to $32 \%$ of $4-\mathrm{H}$ families had two or more computers in the home with the newest computer being purchased in 2004 (26.3\%) see Table 2. Most (93.8\%) of computers systems had a CD-ROM, however, less than half (49\%) had a DVD drive see Table 3.

Table 1

Current Operating System

\begin{tabular}{|l|r|r|r|r|r|r|r|r|}
\hline \multicolumn{10}{|c|}{ Operating System } & \multicolumn{1}{c|}{$\begin{array}{c}\text { No } \\
\text { Computer }\end{array}$} \\
\hline Count & WinXP & \multicolumn{1}{|c|}{ Win98 } & Win2000 & WinME & Mac OSX & Other & Mac OS9 & 1 \\
Percent & 257 & 98 & 47 & 33 & 8 & 2 & 1 & 1 \\
\hline
\end{tabular}


Table 2

Year newest computer obtained

\begin{tabular}{|l|r|r|r|r|r|r|r|r|}
\hline \multicolumn{8}{|c|}{ Year the newest computer was obtained } \\
\hline & None & 2005 & 2004 & 2003 & 2002 & 2001 & 2000 & \multicolumn{1}{c|}{$\begin{array}{c}\text { Before } \\
\end{array}$} \\
\hline Count & 1 & 54 & 119 & 89 & 61 & 36 & 46 & 48 \\
Percent & $.2 \%$ & $11.9 \%$ & $26.3 \%$ & $19.6 \%$ & $13.5 \%$ & $7.9 \%$ & $10.2 \%$ & $10.6 \%$ \\
\hline
\end{tabular}

Table 3

Computer Components

\begin{tabular}{|c|c|c|c|c|c|c|c|c|c|c|c|c|c|c|c|c|}
\hline \multicolumn{17}{|c|}{ Does your primary computer have the following } \\
\hline & \multicolumn{2}{|c|}{ CD-ROM } & \multicolumn{2}{|c|}{ DVD } & \multicolumn{2}{|c|}{$\begin{array}{c}\text { CD-ROM } \\
\text { Burner }\end{array}$} & \multicolumn{2}{|c|}{ DVD Burner } & \multicolumn{2}{|c|}{ USB } & \multicolumn{2}{|c|}{ Firewire } & \multicolumn{2}{|c|}{ AGP } & \multicolumn{2}{|c|}{ Wireless } \\
\hline & No & Yes & No & Yes & No & Yes & No & Yes & No & Yes & No & Yes & No & Yes & No & Yes \\
\hline Count & 28 & 427 & 232 & 223 & 154 & 301 & 351 & 104 & 151 & 304 & 360 & 94 & 357 & 97 & 395 & 59 \\
\hline Percent & 6.2 & 93.8 & 51 & 49 & 33.8 & 66.2 & 77.1 & 22.9 & 33.2 & 66.8 & 79.3 & 20.7 & 78.6 & 21.4 & 87 & 13 \\
\hline
\end{tabular}

\section{Internet Access}

Overall, $8.4 \%$ of respondents did not have access to the Internet. Most (53.1\%) used a dial-up account; $20.9 \%$ had a DSL connection while $8.4 \%$ and $5.7 \%$ had cable and satellite connections respectively as shown in Table 4. A majority of respondents (51\%) do not have high speed access. The most likely barriers to high speed access included costs $(31.3 \%)$ and availability (16.4\%) see Table 6 . When connecting to the Internet, most respondents use Internet Explorer (85.9\%) followed by Netscape Navigator (16.2\%) and Mozilla Firefox (6.3\%).

Table 4

Internet Access

\begin{tabular}{|l|r|r|r|r|r|r|}
\hline \multicolumn{7}{|c|}{ Do you currently access the Internet using } \\
\hline & Dial-up & DSL & $\begin{array}{c}\text { No } \\
\text { Access }\end{array}$ & Cable & Satellite & Other \\
\hline $\begin{array}{l}\text { Count } \\
\text { Percent }\end{array}$ & 241 & 95 & 42 & 38 & 26 & 12 \\
\cline { 2 - 7 } & $53.1 \%$ & $20.9 \%$ & $9.3 \%$ & $8.4 \%$ & $5.7 \%$ & $2.6 \%$ \\
\hline
\end{tabular}

Table 5

High Speed Internet Access

\begin{tabular}{|l|r|r|r|}
\hline \multicolumn{4}{|c|}{ Do you have high-speed Internet access } \\
\hline & No internet access & \multicolumn{1}{|c|}{ Yes } & \multicolumn{1}{|c|}{ No } \\
\hline Count & 38 & 185 & 232 \\
\hline Percent & $8.4 \%$ & $40.7 \%$ & $51.0 \%$ \\
\hline
\end{tabular}

Table 6

Reasons for not having high-speed access

\begin{tabular}{|l|r|r|r|r|r|r|r|}
\hline \multicolumn{10}{|c|}{ Reason for not having high-speed Internet } \\
\hline & Costs & $\begin{array}{l}\text { Have } \\
\text { high- } \\
\text { speed }\end{array}$ & $\begin{array}{c}\text { Not } \\
\text { available }\end{array}$ & $\begin{array}{c}\text { Don't } \\
\text { need }\end{array}$ & $\begin{array}{c}\text { Use } \\
\text { elsewhere }\end{array}$ & Other & $\begin{array}{c}\text { Privacy } \\
\text { and } \\
\text { Security }\end{array}$ \\
\hline Count & 149 & 137 & 78 & 26 & 15 & 6 & 5 \\
Percent & $35.8 \%$ & $32.9 \%$ & $18.8 \%$ & $6.3 \%$ & $3.6 \%$ & $1.4 \%$ & $1.2 \%$ \\
\hline
\end{tabular}




\section{Computer Use}

The primary reported uses of the computer in the home were school related (82.2\%), Email use $(79.8 \%)$, work related (57.1\%) and games (50.1\%) (Table 7$)$. Other or secondary uses of the computer reported were: word processing (88.3\%), Email $(87.7 \%)$, and spreadsheet /database use $(57.5 \%)$ (Table 8 ). Finally, $65.5 \%$ of the respondents indicated they had a digital camera and $24.2 \%$ indicated they had a digital video camera in the household.

Table 7

Primary computer use

\begin{tabular}{|l|r|r|r|r|r|r|r|r|r|r|r|r|}
\hline \multicolumn{10}{|c|}{ Primary use of the computer at home } \\
\hline & \multicolumn{1}{|c|}{$\begin{array}{c}\text { School - } \\
\text { homework }\end{array}$} & \multicolumn{2}{c|}{ Email } & \multicolumn{2}{c|}{ Work } & \multicolumn{2}{c|}{ Games } & \multicolumn{2}{c|}{ Other } & \multicolumn{3}{c|}{ No } \\
& \multicolumn{1}{|c|}{ Computer } \\
& No & Yes & No & Yes & No & Yes & No & Yes & No & Yes & No & Yes \\
\hline Count & 81 & 374 & 92 & 363 & 195 & 260 & 227 & 228 & 407 & 48 & 454 & 1 \\
Percent & 17.8 & 82.2 & 20.2 & 79.8 & 42.9 & 57.1 & 49.9 & 50.1 & 89.5 & 10.5 & 99.8 & 0.2 \\
\hline
\end{tabular}

Table 8

Other uses of computer at Home

\begin{tabular}{|c|c|c|c|c|c|c|c|c|c|c|c|c|c|c|}
\hline \multicolumn{15}{|c|}{ Other uses of the computer at home } \\
\hline & \multicolumn{2}{|c|}{$\begin{array}{c}\text { Word } \\
\text { processing }\end{array}$} & \multicolumn{2}{|c|}{ Email } & \multicolumn{2}{|c|}{$\begin{array}{l}\text { Spreadsheets } \\
\text { and database }\end{array}$} & \multicolumn{2}{|c|}{$\begin{array}{c}\text { Manipulate } \\
\text { graphics and } \\
\text { video }\end{array}$} & \multicolumn{2}{|c|}{ Web Pages } & \multicolumn{2}{|c|}{ Programming } & \multicolumn{2}{|c|}{ Other } \\
\hline & No & Yes & No & Yes & No & Yes & No & Yes & No & Yes & No & Yes & No & Yes \\
\hline Count & 53 & 401 & 56 & 398 & 193 & 261 & 326 & 128 & 336 & 118 & 418 & 36 & 411 & 43 \\
\hline Percent & 11.7 & 88.3 & 12.3 & 87.7 & 42.5 & 57.5 & 71.8 & 28.2 & 74.0 & 26.0 & 92.1 & $7.9 \%$ & 90.5 & 9.5 \\
\hline
\end{tabular}

Note: One respondent indicated they did not have a computer for $0.2 \%$.

For those respondents that reported that they did not have a computer, $2.4 \%$ indicated the reason was that costs were too high while $0.9 \%$ indicated they could use a computer at another location (Table 9).

Table 9

Main reasons for not having a computer at home

\begin{tabular}{|l|r|r|r|r|}
\hline \multicolumn{5}{|c|}{ Main reason for not having a computer } \\
\hline & $\begin{array}{c}\text { Have a } \\
\text { computer }\end{array}$ & $\begin{array}{c}\text { Costs are too } \\
\text { high }\end{array}$ & $\begin{array}{c}\text { Can use } \\
\text { elsewhere }\end{array}$ & \multicolumn{1}{c|}{ Other } \\
\hline Count & 442 & 11 & 4 & 3 \\
Row N \% & $96.1 \%$ & $2.4 \%$ & $.9 \%$ & $.7 \%$ \\
\hline
\end{tabular}

\section{Technology and Science Interest Areas}

Participants were asked to indicate their interests' in areas of technology. The interest areas were scored on a 4-point Likert-type system where NOT = 1, LOW = 2, MEDIUM = 3, and $\mathrm{HIGH}=4$. Overall, the technology areas with the highest mean scores were basic computer knowledge and office applications where the mean scores were 3.47 and 3.42 respectively (Table 10). The results are also broken down by districts. In Nebraska there are four districts: the Northeast (NE), Southeast (SE), Panhandle (PH) and West Central (WC). Not surprisingly, when asked to rank the most important development areas, overall, 58\% selected the basic computer knowledge area, followed by office applications (44\%) and graphic arts (17\%) (Tables 11-13). 
Table 10

Technology Interest areas by district and total

\begin{tabular}{|c|c|c|c|c|c|c|c|c|c|c|}
\hline District & & $\begin{array}{c}\text { Basic } \\
\text { Computer } \\
\text { Knowledge } \\
\end{array}$ & $\begin{array}{l}\text { Web } \\
\text { Sites }\end{array}$ & $\begin{array}{c}\text { Office } \\
\text { Application }\end{array}$ & $\begin{array}{c}\text { Graphic } \\
\text { Arts } \\
\end{array}$ & $\begin{array}{c}\text { Digital Movie } \\
\text { Creation }\end{array}$ & $\begin{array}{c}\text { Computer } \\
\text { programming }\end{array}$ & Network & GIS/GPS & Robots \\
\hline \multirow[t]{3}{*}{ NE } & $\underline{M}$ & 3.43 & 2.79 & 3.37 & 2.93 & 2.63 & 2.76 & 2.69 & 2.50 & 2.19 \\
\hline & $\underline{\mathrm{n}}$ & 135 & 134 & 133 & 134 & 134 & 133 & 132 & 124 & 127 \\
\hline & $\underline{\mathrm{SD}}$ & .833 & .716 & .764 & .717 & .753 & .780 & .783 & .781 & .774 \\
\hline \multirow[t]{3}{*}{$\mathrm{PH}$} & $\underline{M}$ & 3.62 & 2.79 & 3.50 & 3.00 & 2.60 & 3.00 & 2.91 & 2.69 & 2.40 \\
\hline & $\underline{\mathrm{n}}$ & 58 & 57 & 58 & 57 & 57 & 57 & 57 & 55 & 53 \\
\hline & $\underline{\mathrm{SD}}$ & .721 & .901 & .731 & .802 & .863 & .866 & .851 & .879 & .840 \\
\hline \multirow[t]{3}{*}{ SE } & $\underline{M}$ & 3.46 & 2.75 & 3.42 & 2.99 & 2.63 & 2.67 & 2.68 & 2.51 & 2.38 \\
\hline & $\underline{n}$ & 197 & 201 & 200 & 199 & 200 & 198 & 197 & 170 & 185 \\
\hline & $\underline{\mathrm{SD}}$ & .817 & .805 & .697 & .703 & .829 & .878 & .873 & .885 & .820 \\
\hline \multirow[t]{3}{*}{ WC } & $\underline{\mathrm{M}}$ & 3.43 & 2.86 & 3.45 & 3.11 & 2.74 & 2.73 & 2.88 & 2.73 & 2.66 \\
\hline & $\underline{\mathrm{n}}$ & 70 & 71 & 71 & 70 & 70 & 71 & 69 & 62 & 64 \\
\hline & $\underline{S D}$ & .827 & .867 & .789 & .772 & .912 & .878 & .883 & .908 & .859 \\
\hline \multirow[t]{3}{*}{ Total } & $\underline{M}$ & 3.47 & 2.78 & 3.42 & 2.99 & 2.64 & 2.74 & 2.73 & 2.56 & 2.36 \\
\hline & $\underline{\mathrm{n}}$ & 465 & 468 & 467 & 465 & 466 & 464 & 460 & 416 & 434 \\
\hline & $\underline{S D}$ & .809 & .800 & .732 & .731 & .824 & .856 & .855 & .865 & .829 \\
\hline
\end{tabular}

Table 11

Percentage break down of those areas ranked MOST important technology area.

\begin{tabular}{|l|r|r|r|r|r|r|r|r|r|r|}
\hline \multicolumn{10}{|c|}{ Most important area } \\
\hline & $\begin{array}{c}\text { Basic } \\
\text { Computer } \\
\text { Knowledge }\end{array}$ & $\begin{array}{c}\text { Office } \\
\text { Application }\end{array}$ & Missing & $\begin{array}{c}\text { Web } \\
\text { Sites }\end{array}$ & $\begin{array}{c}\text { Graphic } \\
\text { Arts }\end{array}$ & Program & $\begin{array}{c}\text { Digital } \\
\text { Movie } \\
\text { Creation }\end{array}$ & Robots & GIS/GPS & Network \\
\hline Count & 278 & 62 & 38 & 31 & 28 & 10 & 10 & 7 & 7 & 5 \\
Percent & $58.4 \%$ & $13.0 \%$ & $8.0 \%$ & $6.5 \%$ & $5.9 \%$ & $2.1 \%$ & $2.1 \%$ & $1.5 \%$ & $1.5 \%$ & $1.1 \%$ \\
\hline
\end{tabular}

Table 12

Percentage break down of those areas ranked SECOND MOST important technology area.

\begin{tabular}{|l|r|r|r|r|r|r|r|r|r|r|}
\hline \multicolumn{10}{|c|}{ Second important area } \\
\hline & $\begin{array}{c}\text { Office } \\
\text { Application }\end{array}$ & $\begin{array}{c}\text { Web } \\
\text { Sites }\end{array}$ & Missing & $\begin{array}{c}\text { Graphic } \\
\text { Arts }\end{array}$ & $\begin{array}{c}\text { Basic } \\
\text { Computer } \\
\text { Knowledge }\end{array}$ & Program & $\begin{array}{c}\text { Digital } \\
\text { Movie } \\
\text { Creation }\end{array}$ & Network & GIS/GPS & Robots \\
\hline Count & 210 & 63 & 40 & 40 & 34 & 30 & 23 & 15 & 14 & 5 \\
Percent & $44.3 \%$ & $13.3 \%$ & $8.4 \%$ & $8.4 \%$ & $7.2 \%$ & $6.3 \%$ & $4.9 \%$ & $3.2 \%$ & $3.0 \%$ & $1.1 \%$ \\
\hline
\end{tabular}

Table 13

Percentage break down of those areas ranked THIRD MOST important technology area.

\begin{tabular}{|c|c|c|c|c|c|c|c|c|c|c|}
\hline \multicolumn{11}{|c|}{ Third important area } \\
\hline & $\begin{array}{c}\text { Graphic } \\
\text { Arts }\end{array}$ & $\begin{array}{l}\text { Web } \\
\text { Sites }\end{array}$ & Program & Missing & $\begin{array}{c}\text { Office } \\
\text { Application }\end{array}$ & Network & $\begin{array}{c}\text { Digital } \\
\text { Movie } \\
\text { Creation } \\
\end{array}$ & GIS/GPS & $\begin{array}{c}\text { Basic } \\
\text { Computer } \\
\text { Knowledge }\end{array}$ & Robots \\
\hline Count & 210 & 63 & 40 & 40 & 34 & 30 & 23 & 15 & 14 & 5 \\
\hline Percent & $44.3 \%$ & $13.3 \%$ & $8.4 \%$ & $8.4 \%$ & $7.2 \%$ & $6.3 \%$ & $4.9 \%$ & $3.2 \%$ & $3.0 \%$ & $1.1 \%$ \\
\hline
\end{tabular}


The science interest areas with the highest mean scores were Environment Sciences, Botany, and Zoology, where the mean scores were 3.44, 3.42 and 3.33 respectively (Table 14). Overall, Environmental Sciences were ranked the most important development area by $25 \%$ of the respondents followed by Botany (21\%) and Botany/Zoology (15\%) (Tables 15-17). Again, the ranking for priority science development areas follows the ranking of science interest areas with Environmental Sciences ranked number one, followed by Botany and Zoology.

Table 14

Science Interest areas by district and total

\begin{tabular}{|c|c|c|c|c|c|c|c|c|c|}
\hline District & & $\begin{array}{c}\text { Biochemistry } \\
\text { (Molecular } \\
\text { biology, } \\
\text { photosynthesis, } \\
\text { food chemistry) }\end{array}$ & $\begin{array}{c}\text { Botany } \\
\text { (Agronomy, } \\
\text { horticulture, } \\
\text { forestry, } \\
\text { plant } \\
\text { taxonomy, } \\
\text { plant } \\
\text { physiology) } \\
\end{array}$ & $\begin{array}{l}\text { Chemistry } \\
\text { (Physical } \\
\text { chemistry, } \\
\text { organic } \\
\text { chemistry } \\
\text { pesticides, } \\
\text { soil } \\
\text { chemistry }\end{array}$ & $\begin{array}{l}\text { Earth and } \\
\text { Space Sciences } \\
\text { (Geology, } \\
\text { meteorology, } \\
\text { geography, } \\
\text { topography, } \\
\text { mineralogy, } \\
\text { archaeology) }\end{array}$ & $\begin{array}{c}\text { Engineering } \\
\text { (Civil, } \\
\text { mechanical, } \\
\text { aeronautical } \\
\text {, electrical, } \\
\text { bioengineeri } \\
\text { ng, lasers) } \\
\end{array}$ & $\begin{array}{c}\text { Environmental } \\
\text { Sciences }\end{array}$ & $\begin{array}{c}\text { Physics } \\
\text { (Solid } \\
\text { state, } \\
\text { optics, } \\
\text { acoustics, } \\
\text { fluid and } \\
\text { gas } \\
\text { dynamics } \\
\text {, }\end{array}$ & $\begin{array}{c}\text { Zoology } \\
\text { (Animal } \\
\text { genetics, } \\
\text { entomology, } \\
\text { animal ecology, } \\
\text { anatomy, } \\
\text { paleontology) }\end{array}$ \\
\hline \multirow[t]{3}{*}{$\mathrm{NE}$} & $\underline{\mathrm{M}}$ & 2.91 & 3.31 & 3.12 & 3.02 & 2.99 & 3.46 & 2.80 & 3.24 \\
\hline & $\underline{n}$ & 125 & 128 & 127 & 127 & 128 & 129 & 127 & 128 \\
\hline & $\underline{\mathrm{SD}}$ & .730 & .612 & .662 & .672 & .748 & .612 & .749 & .661 \\
\hline \multirow[t]{3}{*}{$\mathrm{PH}$} & $\underline{M}$ & 2.96 & 3.47 & 3.09 & 3.22 & 3.25 & 3.40 & 2.80 & 3.47 \\
\hline & $\underline{n}$ & 53 & 55 & 55 & 55 & 55 & 55 & 55 & 55 \\
\hline & $\underline{\mathrm{SD}}$ & .831 & .716 & .800 & .809 & .865 & .784 & .826 & .742 \\
\hline \multirow[t]{3}{*}{ SE } & $\underline{M}$ & 2.89 & 3.45 & 3.17 & 3.17 & 3.07 & 3.43 & 2.74 & 3.30 \\
\hline & $\underline{\mathrm{n}}$ & 192 & 193 & 193 & 193 & 194 & 192 & 193 & 193 \\
\hline & $\underline{\mathrm{SD}}$ & .743 & .585 & .651 & .656 & .749 & .660 & .767 & .693 \\
\hline \multirow[t]{3}{*}{ WC } & $\underline{M}$ & 3.09 & 3.54 & 3.16 & 3.36 & 3.01 & 3.49 & 2.85 & 3.48 \\
\hline & $\underline{n}$ & 67 & 68 & 68 & 67 & 68 & 67 & 67 & 66 \\
\hline & $\underline{\mathrm{SD}}$ & .883 & .656 & .803 & .667 & .782 & .786 & .744 & .685 \\
\hline \multirow[t]{3}{*}{ Total } & $\underline{M}$ & 2.93 & 3.42 & 3.14 & 3.16 & 3.06 & 3.44 & 2.77 & 3.33 \\
\hline & $\underline{n}$ & 442 & 449 & 448 & 447 & 450 & 448 & 447 & 447 \\
\hline & $\underline{\mathrm{SD}}$ & .775 & .629 & .697 & .690 & .769 & .683 & .764 & .696 \\
\hline
\end{tabular}

Table 15

Percentage break down of those areas ranked MOST important science area.

\begin{tabular}{|l|r|r|r|r|r|r|r|r|r|}
\hline \multicolumn{10}{|c|}{ Most important area } \\
\hline & Environment & Botany & Missing & Zoology & Earth/Space & Biochemistry & Engineering & Chemistry & Physics \\
\hline Count & 118 & 90 & 73 & 66 & 33 & 33 & 30 & 24 & 7 \\
Percent & $24.9 \%$ & $19.0 \%$ & $15.4 \%$ & $13.9 \%$ & $7.0 \%$ & $7.0 \%$ & $6.3 \%$ & $5.1 \%$ & $1.5 \%$ \\
\hline
\end{tabular}

Table 16

Percentage break down of those areas ranked SECOND MOST important technology area.

\begin{tabular}{|l|r|r|r|r|r|r|r|r|r|}
\hline \multicolumn{10}{|c|}{ Second important area } \\
\hline \\
\hline Count & Botany & Environment & Missing & Zoology & Earth/Space & Chemistry & Engineering & Biochemistry & Physics \\
\hline Percent & 101 & 77 & 74 & 69 & 48 & 42 & 29 & 25 & 9 \\
& $21.3 \%$ & $16.2 \%$ & $15.6 \%$ & $14.6 \%$ & $10.1 \%$ & $8.9 \%$ & $6.1 \%$ & $5.3 \%$ & $1.9 \%$ \\
\hline
\end{tabular}


Table 17

Percentage break down of those areas ranked THIRD MOST important technology area.

\begin{tabular}{|l|r|r|r|r|r|r|r|r|r|}
\hline \multicolumn{10}{|c|}{ Second important area } \\
\hline \\
\cline { 2 - 2 } & Missing & Botany & Zoology & Environment & Earth/Space & Chemistry & Engineering & Biochemistry & Physics \\
\hline Count & 83 & 74 & 71 & 59 & 52 & 45 & 42 & 24 & 23 \\
Percent & $17.5 \%$ & $15.6 \%$ & $15.0 \%$ & $12.5 \%$ & $11.0 \%$ & $9.5 \%$ & $8.9 \%$ & $5.1 \%$ & $4.9 \%$ \\
\hline
\end{tabular}

\section{Limitations and Implementation for Practice and Research}

Initially, it would appear that a limitation of this study was the low response rate. However, every effort was made to increase the response rate by sending out a pre survey notice and three follow-up reminders to non-respondents (Mangione, 1995; Salant \& Dillman, 1994). Furthermore, participants were selected from a true random sampling of the population being studied, thereby increasing the statistical likelihood that the sample represents the population (Mangione, 1995).

Moreover, two techniques, comparing early to late responders and comparing initial-round responses to second-round responses, were employed to control for the non-response error and no significant differences were found (Linder, Murphy \& Briers, 2001; Linder \& Wingenbach, 2002). In addition, the findings of this survey are in-line with the U.S Census (2003) findings that 83.4 percent of families with children enrolled in grades $\mathrm{K}-12$ have a computer at home.

This study asked three main questions:

- the percentage of Nebraska 4-H families with a computer at home,

- if 4-H families have access to the Internet, and

- to examine areas of interest in technology and science.

First, over 96 percent of Nebraska 4-H families have access to computers at home. In addition, a majority of these systems were less than three years old. Secondly, nearly 92 percent of families had a connection to the Internet from their home with a majority of families using dialup connections. Finally, families are interested in technology programs focused on basic computer knowledge and office application. In science, $4-\mathrm{H}$ families indicated environment sciences and botany were areas of interest.

The results of this study suggest that 4-H families in Nebraska have adequate computer technologies in their homes to take advantage of computer-based, on-line 4-H programs. The results also indicate that 4-H programs can be delivered over the Internet but that download speeds may be an issue with a majority of households using a low bandwidth dial-up connection. Therefore, hybrid delivery systems that utilize multiple technologies to deliver mediated content may be considered as an alternative delivery solution. A hybrid system would allow large media files to be delivered via a CD-ROM while linked to smaller media files that can be easily delivered on-line. Additionally, the use of DVD's may not be an appropriate delivery solution due to the slow adoption of DVD drives in home computer systems.

\section{Conclusion}

Due to the difference in demographics between states, the findings of this study cannot be generalized to the entire population of $4-\mathrm{H}$ families in the country. However, the findings suggest trends in the adaptation of technology by Nebraska 4- $\mathrm{H}$ families thereby providing 
directions for the Nebraska 4-H science and technology programs and the consideration of electronic delivery methods.

Additional studies are needed to clarify the results of this survey; especially in regards to comparing technologies at home in the rural areas of Nebraska with those in more densely populated areas of Nebraska. For the present, these findings suggest that no significant technological barriers exist in the homes of Nebraska 4-H families regarding 4-H science and technology programs and mediated delivery methods and that less than $9 \%$ of families would be currently excluded from participating in programs that required computers and Internet access at home.

These findings can also offer other youth agencies, serving rural populations, a method for obtaining household technology information and the demographics of the populations they serve. This type of information can provide means for new development in programming, curriculum, and communications.

\section{References}

Day, J.C., Janus, A., \& Davis, J. (2003). Computer and Internet Use in the United States: 2003. U.S. Census Bureau . On-line: http://www.census.gov/prod/2005pubs/p23-208.pdf

Lindner, J. R., Murphy, T. H., \& Briers, G. E. (2001). Handling nonresponse in social science research. Journal of Agricultural Education, 42(4), 4353.

Lindner, J. R., \& Wingenbach, G. J. (2002). Communicating the handling of nonresponse error in research in brief articles. Journal of Extension 40 (6). On-line:

http://www.joe.org/joe/2002december/rb1.shtm/

Mangione, T.W. (1995). Mail Surveys: Improving the Quality. Thousand Oaks, CA: Sage Publications.

Salant, P., \& Dillman, D. A. (1994). How to conduct your own survey. New York, NY: John Wiley \& Sons.

The National 4-H Strategic Directions Team (2001). The power of youth in a changing world: The national 4-H strategic plan. On-line: http://www.national4-hheadquarters.gov/library/summary.pdf

(C) Copyright of Journal of Youth Development Bridging Research and Practice. Content may not be copied or emailed to multiple sites or posted to a listserv without copyright holder's express written permission. Contact Editor at: patricia.dawson@oregonstate.edu for details. However, users may print, download or email articles for individual use.

ISSN 2325-4009 (Print); ISSN 2325-4017 (Online) 